\title{
Molecular dynamics simulation of the effect of dislocations on the martensitic transformations in a two-dimensional model
}

\author{
S. V. Dmitriev ${ }^{1,2}$, M. P. Kashchenko ${ }^{3,4}$, J. A. Baimova ${ }^{1,5, \dagger}$, R. I. Babicheva ${ }^{6}$, \\ D. V. Gunderov ${ }^{7,8}$, V. G. Pushin ${ }^{5}$ \\ †julia.a.baimova@gmail.com \\ ${ }^{1}$ Institute for Metals Superplasticity Problems of RAS, Khalturina St. 39, Ufa 450001, Russia \\ ${ }^{2}$ National Research Tomsk State University, Lenin Prosp. 36, Tomsk 634050, Russia \\ ${ }^{3}$ Ural Federal University, Mira St. 19, Yekaterinburg 620002, Russia \\ ${ }^{4}$ Ural State Forest Engineering University, Sibirsky Trakt 37, Yekaterinburg 620100, Russia \\ ${ }^{5}$ M.N. Miheev Institute of Metal Physics of Ural Branch of RAS, Kovalyevskoy St. 18, Yekaterinburg 620990, Russia \\ ${ }^{6}$ Nanyang Technological University, 50 Nanyang Ave, Singapore 639798, Singapore \\ ${ }^{7}$ Institute of Molecule and Crystal Physics, Ufa Research Center RAS, Oktyabrya Prosp. 151, Ufa 450075, Russia \\ ${ }^{8}$ Saint Petersburg State University, Universitetsky Prosp. 28, Saint Petersburg 198504, Russia
}

Investigation of the thermoelastic martensitic transformation is of high interest nowadays because of the numerous applications of the materials with such structural peculiarities. Thermodynamics, kinetics, structure, morphology of martensitic transformation still remain unclear in many respects. From this point of view, the effective way to study various properties of metallic crystals on atomistic level is molecular dynamics simulation, for which good qualitative agreement with the experiment can be achieved even with simple Morse or Lennard-Jones interatomic potentials. In this paper, the effect of dislocations on the direct and reverse martensitic transformation is studied by molecular dynamics simulation in a two-dimensional model of the ordered alloy with the $\mathrm{AB}$ stoichiometry. The three dimensional analog to this structure is $\mathrm{B} 2$ superstructure based on bcc lattice, which is characteristic for intermetallic NiTi alloy. It is found, that the dislocations can be considered as the nucleation centers for martensite phase, increasing the temperature of the direct martensitic transformation in comparison with the homogeneous martensitic transformation. The martensite domains found in the structure after transformation and the reverse martensitic transformation takes place in the presence of the domain boundaries, meaning that the austenite nucleates heterogeneously. At the reverse transformation, splitting of perfect dislocations into partials dislocations took place. Thus, it was established in the present study that, on the one hand, dislocations affect the direct martensitic transformation as the nucleation centers, and from the other hand, reverse martensitic transformation changes the dislocation structure of the modeled alloy.

Keywords: martensitic transformation, martensite, austenite, dislocation, ordered alloy, molecular dynamics.

\section{Introduction}

Martensitic transformation (MT) is a cooperative effect, which indicates the existence of a governing process. As a rule, MT has the features of the phase transition of the first order, presence of which is determined by the deviation of the starting temperature of $\mathrm{MT}, T_{\mathrm{MS}}$, from the temperature of phase equilibrium, $T_{0}$. For a large deviation of $T_{\mathrm{MS}}$ from $T_{0}$, the description of all the peculiarities of MT is presented in the frames of the heterogeneous nucleation and controlling wave process. Moreover, controlling wave process is caused by the initial excited (oscillating) state in the unstable austenite phase, localized in the elastic field of dislocation nucleation center (DNC) [1].

For small deviations of $T_{\mathrm{MS}}$ from $T_{0}$, MT is close to phase transitions of the second order, and coexistence of heterogeneous and homogeneous nucleation processes is possible. The most interesting are MTs in alloys with shape memory (SM) effect, in particular, in NiTi based alloys $[2,3]$. As a rule, the $\mathrm{SM}$ effect is associated with thermoelastic MT, which have low values of critical deformations and high coherence of phases, which contributes to reversibility of MT upon heating. To date, thermoelastic MT was studied for industrially important allows: Ti-Ni-Cu systems [4], Ti-Ni-Hf [5], Ni-Mn-Ti alloys [6], Ni-Mn-Ga systems [7, 8] to name a few. Using the ideas about the role of DNC and controlling wave process in calculating the morphological features of MT in alloys with SM effect $[9,10]$, including specific twinning variants [11], leads to good agreement with the experiment. Therefore, the problem of independent consideration of the dynamic mechanism of thermoelastic growth in the SM alloys seems to be relevant. The simplest version is to analyze the formation of transformation twins, while it is desirable to compare cases of homogeneous and heterogeneous nucleation. 
To date, along with experimental studies, an effective method for studying the features of the MT [12-18] and the influence of various factors (grain size [13, 14], deviation of composition from stoichiometric [16], external pressure [18], presence of dislocations [19] ets.) is molecular dynamics (MD) simulation. In [19] authors investigate the effect of dislocations on the bcc-hcp transformation in $\mathrm{Zr}$ and show that the dislocations essentially stimulate the MT. The best way to properly investigate any physical process by $\mathrm{MD}$ is the using of the realistic interatomic potentials [19-21], but simple pair interatomic potentials can also give meaningful results [22-27]. In [22] it was that MD method was repeatedly used to study MTs in a two-dimensional (2D) formulation using simple interatomic potentials, and that the obtained results demonstrate a good qualitative agreement with experiment.

The purpose of this paper is to analyze, within the framework of the MD, the problem of the nucleation of martensite with the help of 2D model of a biatomic crystal, which possesses the minimum necessary set of parameters but reflects the characteristic features of the physical system.

\section{Simulation details}

Computational cell contains 32768 atoms with the $128 \times 128$ translational cells each of which contains two atoms of different types. Periodic boundary conditions are applied along $x$ and $y$ directions. The initial phase is austenite. In Fig. 1, the schematic of high symmetric austenite phase and two martensite phases $M_{1}$ and $M_{2}$ is shown. Martensitic phase $M_{1}$ can be obtained from austenite by the shear of close-packed atomic rows alternatively by $a /(2 \sqrt{2})$ (shown by arrows in Fig. 1a) with the following relaxation, while $\mathrm{M}_{2}$ can be obtained from austenite by the shear of close-packed atomic rows by the value multiple to $a /(2 \sqrt{2})$ (shown by arrows in Fig. 1a). The three-dimensional analog of this phase is B2 superstructure based on bcc lattice, which is characteristic for NiTi intermetallic alloy. Note that the martensite phase $M_{1}$ can be considered as a twinned phase $\mathrm{M}_{2}$.

It should be noted, that in the considered model the deformations related to the reconstruction of cells are large, although this is not typical for thermoelastic MT. An advantage of choosing such model parameters is that for large deformations associated with the transformation identification of different phases in simulations becomes much simpler. In addition, large temperature hysteresis in MT can be expected.

Calculations are carried out for a defect-free cell and cell containing a pair of edge dislocations in a prismatic dislocation loop. The procedure of introducing a prismatic dislocation loop into the computation cell is as following. First, atoms in a horizontal strip with thickness of two atomic layers and length of a half of the computational cell size are removed from the crystal in the center of the computational cell. Then, during the process of the atomic relaxation, the edges of the trench merge, and two perfect edge dislocations appear in the cell.

The home-made program code is used to conduct the MD simulations. The interaction between atoms in the system is described with the Morse potential $\varphi(r)=D\left(e^{-2 \alpha(r-R)}-2 e^{-\alpha(r-R)}\right)$, where $D$ is the energy of bond breaking, $R$ - equilibrium bond length, $\alpha$ - bond rigidity. Morse potential was previously successfully used for the simulation of thermoelastic MT $[12,22]$, vacancy clusters in fcc metals [28], hydrogen impurities in Pd and Ni [29], discrete breathers [30-34] and segregations in 2D crystal [35].

It is important to describe a stable diatomic ordered alloy with the $A B$ stoichiometry. In this case, for the description of the interatomic interaction it is required to find the parameters of three potential functions for $A-A, B-B$ and $A-B$ bonds. The most suitable parameters are $D_{A A}=D_{B B}=1$, $D_{A B}=2, \alpha_{A A}=\alpha_{B B}=\alpha_{A B}=5, R_{A A}=R_{B B}=1$ and $R_{A B}=0.85$, as it was adopted in [12]. The bonding energy is chosen such that the ordered state of the alloy is preferable. Indeed, the ordering energy is $E=D_{A A}+D_{B B}-2 D_{A B}=-2$ and the negative value means that at low temperatures the ordered state would be preferable.

At the initial state, all the atoms have zero velocity and get a random deviations from the equilibrium positions. The amplitude of the initial deviations defines the energy given to the system. The potential $E_{p}$, kinetic $K$ and total $E_{T}$ energy of the system, energy of the phase composition, normal and shear stresses, strain are calculated during $t=300$ dimensionless time units. Kinetic energy, $K$, is considered as the measure of temperature. The time step used for integration of the equation of atomic motion is 0.01 .

Potential energy per atom as the function of $\mathrm{K}$ is plotted in Fig. 1(b). In this simulation, relatively small simulation sell is taken $(20 \times 20$ translational cells $)$ to suppress phase transition. Doing so one able to obtain martensite and austenite phases in the temperature range from 0.18 to 0.21 . It can be seen that the curves cross at about $K=0.18$. For smaller (higher) temperature martensite (austenite) phase is energetically preferable. It is seen, that dependence of $E_{p}$ on $K$ for $\mathrm{M}_{1}$ and $\mathrm{M}_{2}$ is almost the same. It means that both types of martensite can competitively appear during MT, which is defined by the chosen potential parameters.
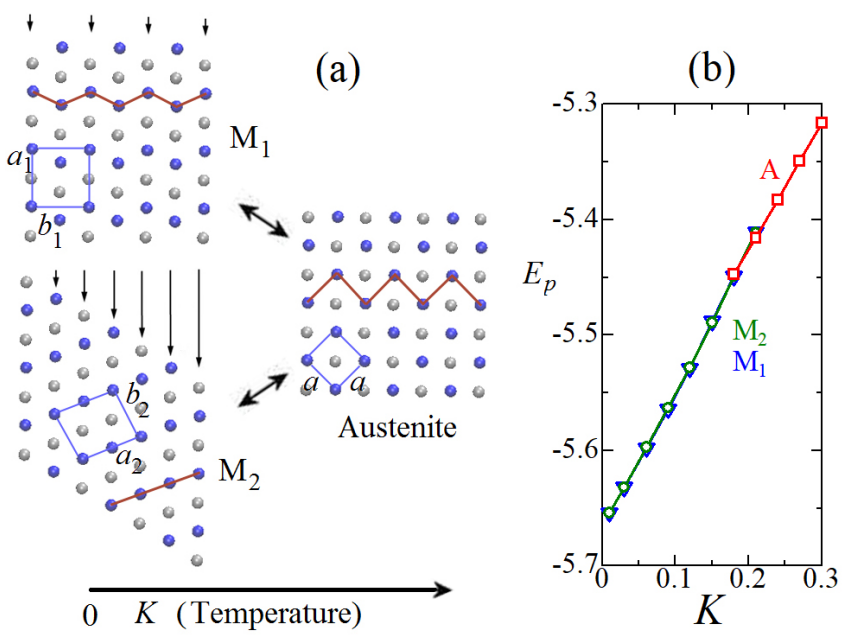

Fig. 1. (Color online) (a) Schematic of phase transition. $M_{1}$ and $M_{2}$ are two types of martensite. (b) Potential energy per atom as the function of kinetic energy (measure of temperature) for martensite $\mathrm{M}_{1}$ (triangles) and $\mathrm{M}_{2}$ (circles), and austenite (squares). 


\section{Results and discussion}

\subsection{Defect-free structure}

In Fig. 2, phase composition of the system as the function of $K$ (temperature) for the direct (solid lines) and the reverse (dashed lines) MT is shown. The starting point for the phase transition is determined by a noticeable change in the slope of the curves that determine the proportion of the austenite and martensite $\left(M_{1}+M_{2}\right)$ phases. The starting temperature for the direct $\mathrm{MT}$ is $K_{\mathrm{MS}} \approx 0.2$, and for the reverse $\mathrm{MT}$ is $K_{\mathrm{AS}} \approx 0.12$. It should be noted that the inequality $K_{\mathrm{MS}}>K_{\mathrm{AS}}$ reflects the presence of wide overlapping temperature ranges for the realization of direct and reverse MT, which can be explained by large values of the elastic strain related with MT. Obviously, $K_{\mathrm{AS}}>K_{\mathrm{MF}}$, where $K_{\mathrm{MF}}$ corresponds to the lower boundary of temperature of the direct MT. From Fig. 2a, it is seen that the number of areas of distorted austenite is maximized when the proportions of austenite and martensite are close. As it follows from Fig. 2b, the fraction of the martensitic phase $M_{1}$ is always greater than fraction of $\mathrm{M}_{2}$.

\subsection{Structure with dislocations}

In Fig. 3 the same results as in Fig. 2 are shown, but for the case when a pair of perfect edge dislocations is introduced to the computational cell. From the comparison of the figures it is seen that the temperatures of the beginning of the direct and reverse transformations slightly shift toward higher temperatures in comparison with the case without dislocations. As it follows from Fig. 3b, the fraction of the martensite $M_{1}$ is always greater than $M_{2}$ phase, but, in comparison with the case without dislocations, the predominance of the $M_{1}$ phase is less pronounced.

Let us discuss the phase distribution in the simulation cell for a direct MT in the absence (Fig. 4) and presence (Fig. 5) of a pair of edge dislocations in the system. Note that in Figs. 4,5, and 6 phase distributions are shown after 300 time units annealing at the constant kinetic energy specified for each panel. Kinetic energy is decreased or increased by steps of 0.2 followed by the annealing. Longer simulation did not produce any noticeable change in the phase distribution. The austenite is shown in red, the distorted regions - in gray, the martensite $M_{1}$ in yellow and $M_{2}$ in dark blue color. All the kinetic energies correspond to the different stages of MT: $(K=0.22)$ initial austenite structure; $(K=0.2)$ appearance of the first martensite plates; $(K=0.18)$ appearance of the numerous crossing martensite plates with some distorted regions between them; $(K=0.16)$ appearance of the martensite twins; $(K=0.14)$ formation of the almost martensite structure; and $(K=0.12)$ final martensite structure. The same stages are shown in Fig. 5 with two schematically shown dislocations.

From the comparison of Fig. 4 and 5, it is seen that in the absence of dislocations at $K=0.22$, there is an ideal austenite phase. In the structure with the dislocations, the appearance of martensite, which has the form of segments (analogues of plates), inclined at angles $\pm 45^{\circ}$ to the horizontal plane, is noted. This corresponds to the orientation of the plates expected on the basis of the concept of controlling wave process [1], when its growth is controlled by a pair of longitudinal waves running with velocities of the same magnitude along the orthogonal symmetry axes of order of four.

Since the remaining segments (plates) of martensite have analogous orientations, it is assumed, that under homogeneous nucleation the character of the initial excited state is the same as for the high-symmetry version of a DNC of purely edge type, which does not violate its elastic field by selecting orientations of wave vectors of longitudinal wave beams, but only reducing the magnitude of the barrier. This predetermines the appearance of the first martensite crystal near the DNC. It is interesting to change the orientation of DNC after which the changing of the orientation of the line corresponding to the orientation of the habitus of the crystal close to the DNC is expected while the conservation of the previous orientation for homogeneous nucleation took place. It should be mentioned that short-wave shifts are considered in the model, the group velocities corresponding to them are small, and in real crystals they are strongly damped.

Dislocations in the system also affects the formation of domains of the martensite phase. Thus, in Fig. 4 domain boundaries (shown in gray because they represent distorted areas) are smoother than in Fig. 5, where they have clearly seen kinks. Residual austenite is observed at fairly low temperatures, up to $K=0.12$, both in the case without and with the dislocations.
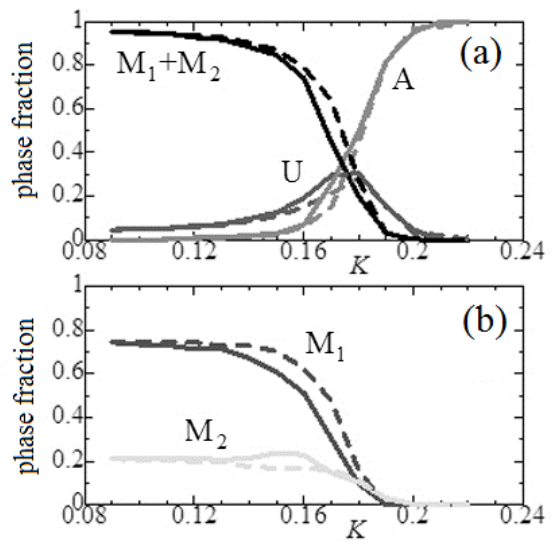

Fig. 2. Structure without dislocations. Phase composition of the system as the function of $\mathrm{K}$ (temperature) for the direct (solid lines) and the reverse (dashed lines) MT. In (a) fractions of the austenite phase (light gray curves), two martensitic phases (black curves) and distorted interphase regions (dark gray curves) are shown. In (b), the fractions of $M_{1}$ and $M_{2}$ are given.

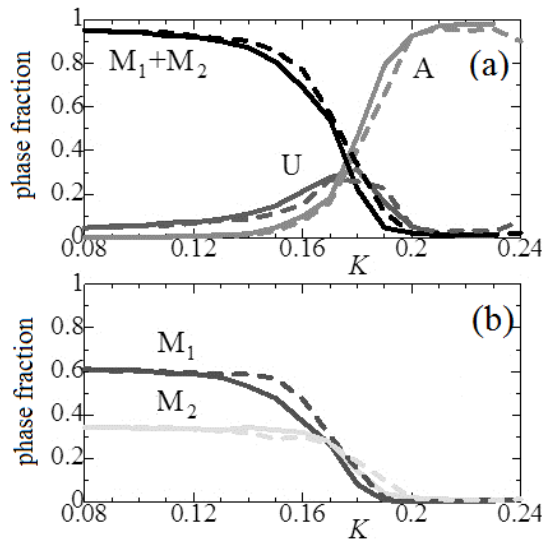

Fig. 3. Same as in Fig. 2, but in the presence of dislocations. 


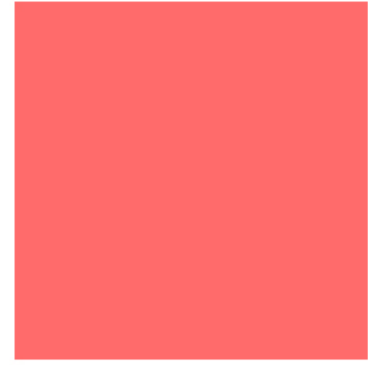

$K=0.22$

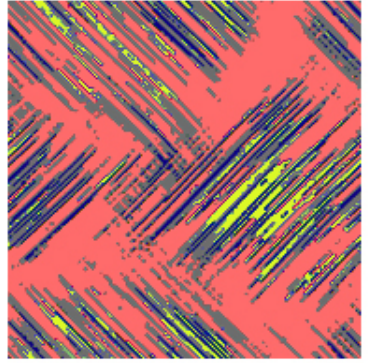

$K=0.18$

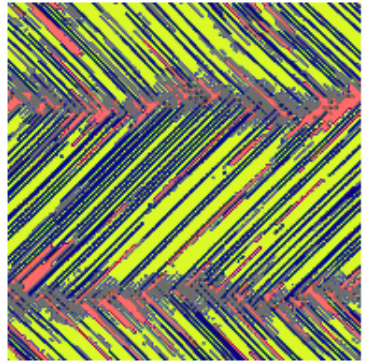

$K=0.16$

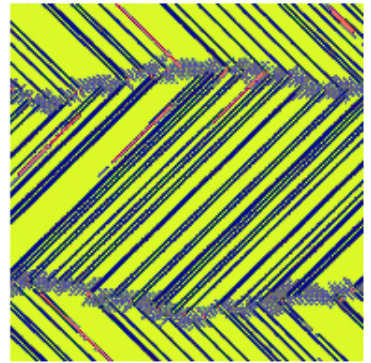

$K=0.12$

Fig. 4. (Color online) Direct (during cooling) MT in the absence of dislocations. The phase distribution is shown for for values of the kinetic energy $K$ (temperature). Here austenite (A) is shown in red (light gray), distorted interphase areas (U) in gray (dark gray), martensite $\mathrm{M}_{1}$ in yellow (white) and $\mathrm{M}_{2}$ in dark blue (black). Colors in brackets are for printed black and white printed version.

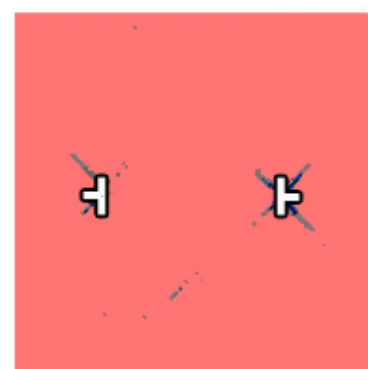

$K=0.22$

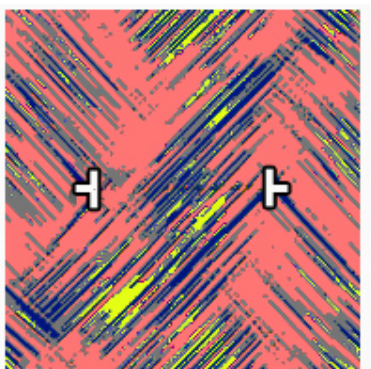

$K=0.18$

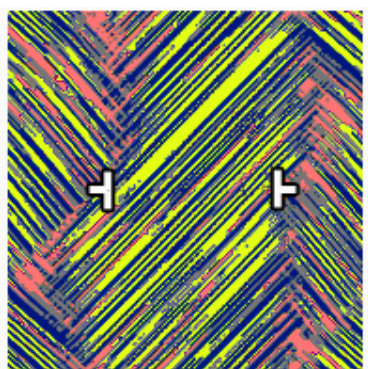

$K=0.16$

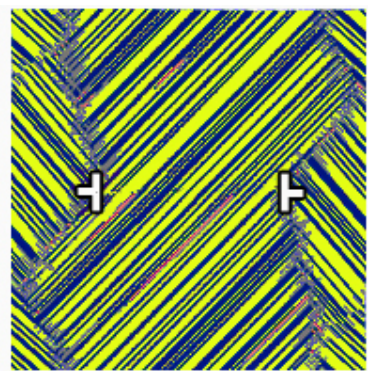

$K=0.12$

Fig. 5. (Color online) Direct (during cooling) MT in the presence of dislocations. Phases are colored as in Fig. 4.

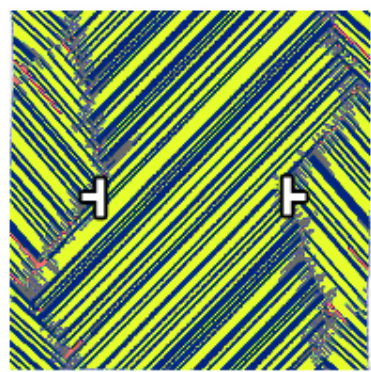

$K=0.12$

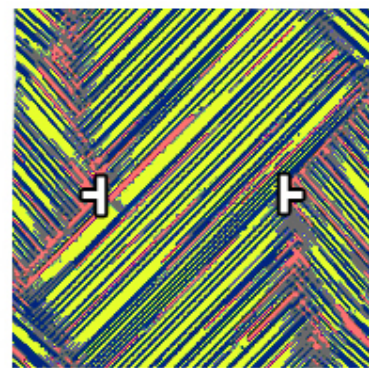

$K=0.16$

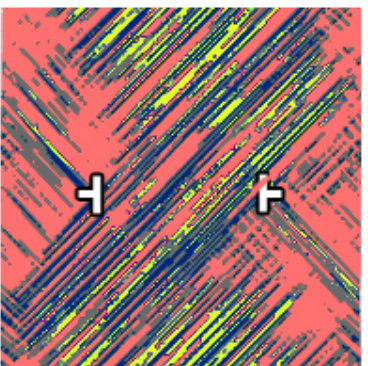

$K=0.18$

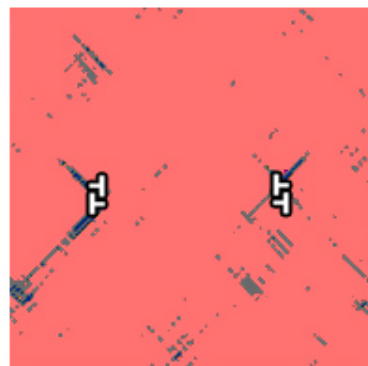

$K=0.22$

Fig. 6. (Color online) The reverse (during heating) MT in the presence of dislocations. Phases are colored as in Fig. 4.

An interesting transformation of dislocations occurs during the reverse MT shown in Fig. 6. The splitting of the initial perfect dislocations into partial dislocations takes place even at $K=0.18$, which becomes especially noticeable at $K=0.20$. According to our analysis, partial dislocations move and at $K=0.22$ the distance between them increases noticeably. Looking at the phase distribution at $K=0.22$ shown in Fig. 6, attention should be paid to the remaining plates of martensite near the dislocations. In the absence of dislocations at $K=0.22$, martensite almost completely disappears.

The above discussion shows that, despite the simplicity of the considered model, the obtained results are in a qualitative agreement with the theoretical predictions [1]. Nevertheless, not all the features of experimentally observable structures are reproduced by this model. In particular, the interaction of the two martensitic phases during transformation, leading to a change of their volume fractions is an unusual feature.

In the future studies of MT with the help of MD, it would be interesting to consider models producing smaller (by one order of magnitude) deformations and with other variants of
DNC. When choosing the level of lattice deformation, it is important to take into account the relative change in volume at MT, which should affect the transformation temperatures. In the presence of DNC, the most interesting problem is the visualization of the appearance of the initial excited (vibrational) state that generates the controlling wave process.

\section{Conclusions}

MD simulation of direct and reverse MT in the framework of the 2D model of an ordered alloy shows that dislocations can be considered as the nucleation centers of martensite during direct MT. In the absence of dislocations, martensite nucleates homogeneously at lower temperatures. The reverse MT occurs in the presence of twinned martensite and domain walls in the system, which means that even in the absence of dislocations, the structure of the alloy is not ideal. During the reverse MT, the perfect dislocations split into partial dislocations. It should be noted that for selected model parameters MT is associated with large lattices deformations, therefore, temperature hysteresis is also large, which is not 
typical for SM alloys. However, the consideration of the effects of SM and superelasticity is not prohibited in frame of this model, since the degree of coherence of the martensite and austenite lattices is quite high. One of the important problems for futher investigations is the studying of the MT as the detonation process [36].

Aknowledgements. S. V. D. acknowledges support from the Russian Foundation for Basic Research, grant No. 16-58-48001 IND_omi (design of the research, discussion of the results). J.A. B and V.G. P. are grateful for the financial support from the Russian Science Foundation, grant No. 15-12-10014 (numerical simulations and writing the paper). R.I. B. thanks financial support from the Russian Science Foundation, grant No. 17-79-10410 (numerical simulations). The work of D. V. G. was supported by the Saint-Petersburg State University, research grant No. 6.65.43.2017 (discussion of the results).

\section{References}

1. M.P. Kashchenko, V.G. Chashchina. Phys. Usp. 54 (4), 331 (2011).

2. V.N. Khachin, V.G. Pushin, V.V. Kondratev. Ed.V. D. Sadovskii. - M.: Nauka, 1992. 161 p. (in Russian) [Хачин В.Н., Пушин В.Г., Кондратьев В.В. Отв. ред. В. Д. Садовский. - М.: Наука, 1992. 161 с.]

3. Shape Memory NiTi Alloys.. Part 1. Structure, Phase Transformation, and Properties. Ed.V. G. Pushin: Ekaterinburg: YrO RAS, 2006. - 414 p. (in Russian) [Сплавы никелида титана с памятью формы. Ч.1. Структура, фазовые превращения и свойства. Под ред. В. Г. Пушина: Екатеринбург: УрО РАН, 2006. 414 c.]

4. N. N. Kuranova, A. V. Pushin, V.G. Pushin, A. V. Korolev, N. I. Kourov. Tech. Phys. Lett. 42, 376 (2016).

5. V.G. Pushin, N.N. Kuranova, A.V. Pushin, A. N. Uksusnikov, and N.I. Kourova. Tech. Phys. 61 (7), 1009 (2016).

6. E.S. Belosludtseva, N.N. Kuranova, N.I. Kourov, V.G. Pushin, A.N. Uksusnikov. Tech. Phys. 60, 1330 (2015).

7. I. I. Musabirov, I. Z. Sharipov, R. R. Mulyukov. Russ. Phys. J. 58 (6), 745 (2015).

8. I.I. Musabirov, I.M. Safarov, R.R. Mulyukov, I.Z. Sharipov, V.V. Koledov. Letters on Materials. 4 (4), 265 (2014).

9. M.P. Kashchenko, V. G. Chashchina. Met. Sci. Heat Treat. 55 (11-12), 643 (2014).

10. M.P. Kashchenko, V. G. Chashchina. Russ. Phys. J. 56 (7), 807 (2013).

11. M. P. Kashchenko, V.G. Chashchina. Phys. Mesomech. 19 (3), 107 (2016).

12. R. I. Babicheva, J. A. Baimova, S. V. Dmitriev, V. G. Pushin. Letters on Materials. 5 (4), 359 (2015).

13. K. R. Morrison, M. J. Cherukara, H. Kim, A. Strachan.
Acta Mater. 95, 37 (2015).

14. C. Ni, H. Ding, X. J. Jin. J. Alloys Compd. 546, 1 (2013).

15. S. Kazanc, F. A. Celik, S. Ozgen. J. Phys. Chem. Solids. 74, 1836 (2013).

16. T. Suzuki, M. Shimno, K. Otsuka, X. Ren, A. Saxena. J. Alloys Compd. 577S, S113 (2013).

17. B. Wang, E. Sak-Saracino, N. Gunkelmann, H. M. Urbassek. Comp. Mater. Sci. 82, 399 (2014).

18. C. Tatar, S. Kazanc. Current Applied Physics. 12, 98 (2012).

19. A.R. Kuznetsov, Yu.N. Gornostyrev, M.I. Katsnelson, A. V. Trefilov. Mater. Sci Eng. A309-310168 (2001).

20. I.N. Kar'kin, Yu. N. Gornostyrev, L.E. Kar'kina. Phys. Solid State. 52, 431 (2010).

21. Y. N. Gornostyrev, I. N. Kar'kin, L.E. Kar'kina. Phys. Solid State. 53, 1388 (2011).

22. J. A. Baimova, R. I. Babicheva, A. V.Lukyanov, V. G. Pushin, D. V. Gunderov, S. V. Dmitriev. Rev. Adv. Mater. Sci. 47, 86 (2016).

23. O. Kastner, G. Eggeler, W. Weiss, G. J. Ackland. J. Mech. Phys. Solids. 59, 1888 (2011).

24. K. Saitoh, W. K. Liu. Comp. Mater. Sci. 46, 531 (2009).

25. T. Suzuki, M. Shimono, S. Takeno. Phys. Rev. Lett. 82, 1474 (1999).

26. O. Kastner. Continuum Mech. Thermodyn. 15, 487 (2003).

27. O. Kastner. Continuum Mech. Thermodyn. 18, 63 (2006).

28. M.D. Starostenkov, M.S. Aksenov, G.M. Poletaev, R.Y. Rakitin. Fundamentalnie problemi sovremennogo materialovedeniya. 3 (2), 16 (2006).

29. G. M. Poletaev, E.S. Medvedeva, I.V. Zorya, D. V. Novoselova, M.D. Starostenkov. Russ. Phys. J. 60 (2), 201 (2017).

30. A. A. Kistanov, R. T. Murzaev, S. V. Dmitriev, V. I. Dubinko, V.V. Khizhnyakov. JETP Letters. 99 (6), 353 (2014).

31. S.V. Dmitriev, E.A. Korznikova, J.A. Baimova, M. G. Velarde. Phys. Usp. 59, 446 (2016).

32. S. V. Dmitriev, A.P. Chetverikov, M.G. Velarde, Phys. Stat. Solidi b 252 (7), 1682 (2015).

33. M.G. Velarde, A.P. Chetverikov, W. Ebeling, S. V. Dmitriev, V.D. Lakhno, European Physical Journal B 89 (10), 233 (2016).

34. A. A. Kistanov, S.V. Dmitriev, A.P. Chetverikov, M. G. Velarde, European Physical Journal B, 87 (9), 5 (2014).

35. R.T. Murzaev, S.V. Dmitriev. Fundamentalnie problemi sovremennogo materialovedeniya. 10 (3), 334 (2013) (in Russian) [Мурзаев P.T., Дмитриев С. В. Фундаментальные проблемы современного материаловедения. 2013. т. 10, № 3, с. 334.]

36. M.I. Alymov, V.S. Trofimov, E.V. Petrov. Letters on Materials 7(1), 26-28 (2017). (in Russian) [Алымов М.И., Трофимов В.С., Петров Е.В. Письма о материалах. 7(1), 26-28 (2017)] DOI: 10.22226/2410-35352017-1-26-28 